\title{
Practice parameters for the use of actigraphy in the military operational context: the Walter Reed Army Institute of Research Operational Research Kit- Actigraphy (WORK-A)
}

Jaime K. Devine ${ }^{1}$, Jake Choynowski ${ }^{2}$, Tina Burke ${ }^{2}$, Kajsa Carlsson ${ }^{2}$, Vincent F. Capaldi ${ }^{2}$, Ashlee B. McKeon ${ }^{2 *}$ and Walter J. Sowden ${ }^{3}$

\begin{abstract}
Background: The Walter Reed Army Institute of Research (WRAIR) Operational Research Kit-Actigraphy (WORK-A) is a set of unique practice parameters and actigraphy-derived measures for the analysis of operational military sleep patterns. The WORK-A draws on best practices from the literature and comprises 15 additional descriptive variables. Here, we demonstrate the WORK-A with a sample of United States Army Reserve Officers' Training Corps (ROTC) cadets $(n=286)$ during a month-long capstone pre-commissioning training exercise.

Methods: The sleep of ROTC cadets $(n=286)$ was measured by Philips Actiwatch devices during the 31-day training exercise. The preliminary effectiveness of the WORK-A was tested by comparing differences in sleep measures collected by Actiwatches as calculated by Philips Actiware software against WORK-A-determined sleep measures and self-report sleep collected from a subset of ROTC cadets $(n=140)$.

Results: Actiware sleep summary statistics were significantly different from WORK-A measures and self-report sleep (all $P \leq 0.001$ ). Bedtimes and waketimes as determined by WORK-A major sleep intervals showed the best agreement with self-report bedtime (22:21 $\pm 1: 30$ vs. $22: 13 \pm 0: 40, P=0.21)$ and waketime (04:30 $\pm 2: 17$ vs. 04:31 \pm 0 : $47, P=0.68)$. Though still significantly different, the discrepancy was smaller between the WORK-A measure of time in bed (TIB) for major sleep intervals ( $352 \pm 29 \mathrm{~min}$ ) and self-report nightly sleep duration (337 $\pm 57 \mathrm{~min}, P=0.006)$ than that between the WORK-A major TIB and Actiware TIB $(177 \pm 42, P \leq 0.001)$.
\end{abstract}

Conclusions: Default actigraphy methods are not the most accurate methods for characterizing soldier sleep, but reliable methods for characterizing operational sleep patterns is a necessary first step in developing strategies to improve soldier readiness. The WORK-A addresses this knowledge gap by providing practice parameters and a robust variety of measures with which to profile sleep behavior in service members.

Keywords: Actigraphy, Sleep-wake patterns, Military sleep assessment, Operational environment, Scoring methodology

\footnotetext{
* Correspondence: ashlee.b.mckeon.mil@mail.mil.

Behavioral Biology Branch, Walter Reed Army Institute of Research, 503 Robert Grant Ave, Silver Spring, MD 20910, USA

Full list of author information is available at the end of the article
}

\section{$\triangle B M C$}

(c) The Author(s). 2020 Open Access This article is licensed under a Creative Commons Attribution 4.0 International License, which permits use, sharing, adaptation, distribution and reproduction in any medium or format, as long as you give appropriate credit to the original author(s) and the source, provide a link to the Creative Commons licence, and indicate if changes were made. The images or other third party material in this article are included in the article's Creative Commons licence, unless indicated otherwise in a credit line to the material. If material is not included in the article's Creative Commons licence and your intended use is not permitted by statutory regulation or exceeds the permitted use, you will need to obtain permission directly from the copyright holder. To view a copy of this licence, visit http://creativecommons.org/licenses/by/4.0/. The Creative Commons Public Domain Dedication waiver (http://creativecommons.org/publicdomain/zero/1.0/) applies to the data made available in this article, unless otherwise stated in a credit line to the data. 


\section{Background}

Service members are more likely than civilian populations to experience sleep disturbances, including shortened sleep, fragmented sleep, and difficulty falling/ maintaining asleep [1-4]. In many ways, these sleep disturbances may closely resemble those seen in shift workers and other operational contexts [5-7]. However, industrial operations have greater capacity to control or document work schedules [8], whereas conditions during military operations are unpredictable [9]. Given these differences in sleep patterns, sleep in the military operational context should be quantified differently than how it is in civilians.

To accurately profile the uniquely erratic sleep patterns of soldiers in the military operational context, researchers on the Operational Research Team (ORT) at the Walter Reed Army Institute of Research (WRAIR) have developed the WRAIR Operational Research Kit-Actigraphy (WORK-A). The WORK-A is a set of actigraphy-derived measures adapted from commercial industry standards for the analysis of sleep patterns that are specific to activeduty soldiers in operational domains.

\section{Actigraphy}

Actigraphy has been a mainstay of sleep research since the late 1970s [10] and been used for the analysis of circadian and rest-activity rhythms (RAR) in healthy and specialty populations [11-16]. Specifically, actigraph devices detect body movement and sum these movements into digital activity counts across a predetermined epoch interval. Actigraphy software infers sleep-wake patterns from activity counts using computerized scoring algorithms that have been validated against polysomnography (PSG). However, while PSG (the gold standard for the measurement of sleep) uses standardized rules for scoring sleep [17], there is no currently established consensus for scoring actigraphy $[18,19]$. Even within actigraphy platforms validated against PSG, such as Philips Actiware [20-22], the quality control of sleep data is left to the interpretation of the researcher.

Supplementary data can help researchers differentiate actual sleep from periods of inactivity or removal of the device. Research actigraphy devices have begun to include additional features such as recording exposure to light, measuring heart rate and temperature, and providing options for the wearer to manually record an event such as bedtime. Newer models can also sense when the device is not being worn and can alert the user to put the watch back on. Apart from the device, research participants will frequently complete self-report diaries during the data collection period which helps add context to the objective sleep/wake schedule of the individual of the individual. However, these supplementary measures may not always be practical, as they increase the burden of data collection on the participant. Characterizing sleep in populations with demanding and irregular schedules may have to rely on stand-alone analysis of actigraphy data without context. Reliable methods of analysis must therefore be established to profile sleep in specific populations, such as active duty military personnel.

Philips default actigraphy settings record one interval of sleep lasting longer than $3 \mathrm{~h}$ per $24 \mathrm{~h}$ period (also called a major sleep interval) and compute average sleep measures (total sleep time [TST], sleep efficiency [SE], wake after sleep onset [WASO], etc.) collected across all sleep intervals. Researchers can adjust the settings to account for minor sleep intervals (between $15 \mathrm{~min}$ to $3 \mathrm{~h}$ in length) and to record more than one sleep interval per day.

However, sleep measures are averaged across all collected sleep intervals without a clear distinction between major and minor sleep intervals. Averaging major and minor sleep intervals together can result in a shorter average sleep duration, even though the individual is receiving more sleep within a $24-\mathrm{h}$ period by taking extra sleep opportunities (i.e., naps). Moreover, measures of sleep quality, such as SE or WASO, are calculated within the bounds of a single sleep interval and thus do not account for fragmentation, due to sleep being split into multiple intervals across the day. It is important to understand how frequently within a 24-h period an individual is sleeping and the length of each sleep interval to accurately estimate average sleep duration and quality.

Standardization of research procedures in actigraphy is progressing. Several critiques and guidelines have been published outlining appropriate actigraphy procedures over the years [18, 23-27]. Moreover, the methodological issues inherent to actigraphy have been addressed for specific populations, such as individuals with sleep disorders [26], nurses [28], cancer patients [19], mothers and infants [29], dementia patients [30] and individuals with traumatic brain injuries [31]. The Society of Behavioral Sleep Medicine and The Journal of Clinical Sleep Medicine recently produced comprehensive instructional manuals for behavioral sleep assessment using actigraphy in general populations [32] and clinical practice [33].

\section{Walter reed Army Institute of research operational research kit-Actigraphy (WORK-A)}

The WORK-A aims to address the problems interpreting actigraphy data outlined above in a population that has not previously been addressed, i.e., military personnel. The primary goal of the WORK-A is to accurately profile soldier sleep rather than rest-activity or circadian rhythm. As such, only measures related to sleep duration and sleep quality or methods to facilitate interpretation of these data were considered for incorporation into the 
kit. The selection for measures to include in this kit was informed by data collected from service member populations as part of the ORT's operational field studies as well as input from sleep research professionals and U.S. Army officers. The WORK-A is designed to apply to a wide range of operational scenarios, but for the purposes of this paper, it has been applied to Reserve Officers' Training Corps (ROTC) cadets undergoing Advanced Camp.

The Cadet Summer Training Advanced Camp at Fort Knox is a 31-day training event designed to assess an ROTC cadets' ability to meet US Army standards through a tiered training structure using light infantry tactics as the instructional medium. ROTC Advanced Camp is the U.S. Army's largest training exercise and the U.S. Army Cadet Command's capstone training event. ROTC Advanced Camp provides a realistic glimpse into the military operational context, thus making it an ideal setting for the initial application of the WORK-A.

Here, we present the methodology for actigraph configuration and pre-processing, the minimum data requirements, and the calculation of variables from processed and exported actigraphy data, which constitute the full research toolkit known as the WORK-A. The toolkit relies on methods that are established in the literature and attempts to minimize novel methodologies or subjective interpretation. To maximize the measuring accuracy of soldier sleep, the WORK-A has been applied to a dataset of activity recordings collected from ROTC cadets undergoing Advanced Camp to assess agreement between traditional measure of actigraphy, the WORK-A and selfreport measures of sleep using Bland-Altman analysis [34, 35], Student's $t$-tests and descriptive statistics.

\section{Methods}

\section{Selection of measured parameters}

The first step in developing the WORK-A was a literature review to determine the most applicable practice parameters for actigraphy, methods for sleep/wake determination for populations with unpredictable schedules, and measures for quantifying sleep. The literature review was conducted using PubMed and Google Scholar search engines using the following combinations of search terms: 1) actigraphy or accelerometry; 2) practice parameters, methods, methodology or practices; and 3) sleep, sleep medicine, sleep research, sleep patterns or sleep/wake behavior. Additional searches were conducted to identify articles applying actigraphy to specific populations that may share common methodological issues with US Army soldiers, such as individuals in specific operational contexts (shift workers, pilots, military, doctors and nurses, long-haul drivers, athletes), individuals with atypical rest-activity rhythms (sleep disordered individuals, circadian disordered patients, parents of infants, etc.), patient populations with comorbid sleep issues (cancer, dementia, mental health disorders) or nonindustrial indigenous groups. Practices and methods from areas outside sleep research/sleep medicine (i.e., circadian misalignment, fragmentation index [36], intradaily variability, interdaily stability, light exposure, RAR, etc.) were not incorporated into the WORK-A. Moreover, not yet validated methods of sleep/wake determination from sleep research were not considered.

While the literature still recommends the use of sleep diaries or other supplementary data to hand score actigraphy, there is reliable confidence in the ability of validated algorithms to accurately measure habitual sleep patterns [19, 26, 27, 32, 33]. By relying on automated scoring algorithms from validated devices with supporting software, the WORK-A can more easily be reproduced by fellow researchers. The literature review also revealed key variables for the reporting of habitual sleep patterns, specifically, measures of habitual bedtime and waketime, time in bed (TIB), sleep onset latency (SOL), TST, WASO and SE [19, 27, 32].

\section{Addressing gaps between sleep measures for civilian versus military populations}

The goal of the WORK-A is not only to quantify military sleep patterns but also to identify how sleep patterns may contribute to military readiness. However, the military operational domain is a variable environment, and soldier sleep patterns can differ greatly based on the context of the mission. Aspects of sleep that may correlate with performance in one population of soldiers (for example, a tank crew) cannot be assumed to be generalizable to a different population (such as Army Rangers). To address this variability, the WORK-A quantifies sleep via the key sleep variables described above (bedtime, wake time, TIB, SOL, TST, WASO, and $\mathrm{SE}$ ) for specific types of sleep (specifically, all sleep intervals, minor sleep intervals, major sleep intervals and daily sleep) to create a versatile toolkit from which different variables can be selected to address research questions relating to a wide range of soldier groups.

\section{Actigraphy measures in WORK-A}

Key actigraphy variables and the WORK-A measures are defined in Table 1. The WORK-A is designed to be adaptable for use with any actigraphy device with adjustments as necessary. Philips Actiware and Actiwatch devices (Actiwatch 2.0, Philips Respironics, Murrysville, PA) were used for the purposes of this paper. Using default settings, Philips Actiware averages key sleep variables (TIB, SOL, TST, WASO, SE) across all recorded sleep intervals to provide a summary statistic. Philips Actiware also provides a mobility measure called the fragmentation index, which is defined as the "sum of 
Table 1 Key actigraphy variables and actigraphy measures in WORK-A

\begin{tabular}{lll}
\hline Metric types & Measure & Definition \\
\hline Key actigraphy variables & Time in bed (TIB) & $\begin{array}{l}\text { The average time elapsed between the start time } \\
\text { and the end time of a given rest interval } \\
\text { (representing time spent in bed/at rest/attempting } \\
\text { to sleep) in minutes }\end{array}$ \\
& $\begin{array}{l}\text { Total sleep time (TST) } \\
\text { ine total number of minutes within a given rest } \\
\end{array}$ & $\begin{array}{l}\text { interval that have been scored as sleep by the } \\
\text { sleep interval (representing time when sleep is } \\
\text { occurring) detection algorithm }\end{array}$
\end{tabular}

Sleep onset latency $(\mathrm{SOL})$

Wake after sleep onset (WASO)

Sleep efficiency (SE)

Actigraphy measures in Walter Reed Army Institute of Research Operational Research Kit-Actigraphy (WORK-A)
All intervals actigraphy measures
Major interval actigraphy measures

Minor interval actigraphy measures

Daily actigraphy measures

Sleep midpoint

Time worn

Daily sleep intervals (DSI)

DSI bedtime (s)

DSI waketime (s)

Soldier sleep below minimum (SSBM)
The time required for sleep to start after initiating the intent to sleep. The time, in minutes, between the start of a given rest interval and the onset of sleep as scored by the sleep interval detection algorithm

The total number of minutes scored as wake within a given sleep interval

The percentage of time spent in bed sleeping. Scored as TST/TIB multiplied by 100

TIB, TST, SOL, WASO and SE averaged across all sleep intervals

TIB, TST, SOL, WASO and SE averaged across only major sleep intervals (sleep intervals lasting longer than $3 \mathrm{~h}$ )

TIB, TST, SOL, WASO and SE averaged across only minor sleep intervals (sleep intervals lasting between $15 \mathrm{~min}$ and $3 \mathrm{~h}$ )

TIB, TST, SOL, WASO and SE combined for all sleep intervals within a 24-h period, then averaged across days worn

Halfway point between the time of sleep onset and final awakening

Number of minutes or days that device was worn

The average number of sleep intervals recorded across all days worn

Average start time for a sleep interval by DSI number within a 24-h period

Average end time for a sleep interval by DSI number within a 24-h period

Number of days during wear that participant slept less than a researcher-specified number of hours percent mobile and percent immobile bouts less than 1 minute in duration to the number of immobile bouts for a given interval". This measure has been applied to sleep intervals to measure sleep fragmentation [37, 38], but it has not been sufficiently utilized in the sleep literature to be considered a key sleep variable $[19,27,32]$. The WORK-A investigated key sleep variables averaged over all sleep intervals, as well as major sleep intervals only, minor sleep intervals only, and overall daily sleep. Daily sleep measures are calculated by combining all sleep intervals from which an individual woke within a 24-h period into a sum for that day. For example, if on a given day, an individual slept for $5 \mathrm{~h}$ and then took a 90min nap, their daily TST would be $6.5 \mathrm{~h}$. Twenty-fourhour periods begin at 12:00 (noon) and end at noon the subsequent day, as per Philips Actiware specifications. Average and median bedtime and wake time, as well as sleep midpoint, were also calculated.

The WORK-A also took into account the time worn and calculates the number of times within a 24-h period (defined as 12:00-12:00) sleep occurs to determine daily sleep intervals (DSI) to account for fragmentation of sleep opportunities. The number of sleep intervals per 24-h period was derived from the DSI based on interval start time. The average bedtime and wake time for the first and second DSI occurring within a day were also included in the WORK-A. Time worn was calculated by summing the number of calendar days during the study period on which the participant wore the watch, subtracting off-wrist days or excluded periods. This ensures 
that days on which no sleep occurred are still accounted for in the data. Next, we averaged the number of sleep intervals per day, start time and end time of intervals (bedtime and waketime) and the length of intervals across the total days the participant wore the actigraph. By doing this, we can more thoroughly describe when and how well soldiers are maximizing their opportunities for sleep.

The final variable in the WORK-A quantifies the soldier as above or below a specified minimum. The National Sleep Foundation recommends 7 or more hours of sleep per night for healthy functioning [39], and while there is currently no Army-mandated minimum sleep requirement [40], the Army has previously recommended that soldiers receive a minimum of $4 \mathrm{~h}$ of sleep within a 24-h period [41]. The soldier sleep below minimum (SSBM) variable can be adjusted to specify a threshold for the minimum acceptable level of sleep (specified for these analyses as daily TST less than $4 \mathrm{~h}$ ) by summating a series of conditional Boolean arguments that categorize daily TST as over $($ false $=0$ ) or under (true $=1$ ) the designated threshold during a specified time period. With this, we can then determine how many days during the study period soldiers fail to meet a specified minimum recommendation for sleep duration, even if they habitually meet the minimum.

The WORK-A also has two additional features to help researchers conceptualize habitual sleep patterns across $24 \mathrm{~h}$. These features calculate a separate dataset with hourly bins of 1) activity counts and 2) the likelihood of a sleep interval occurring during that hour and compiles this information by hour (0000-2300) for each participant within a study sample in a separate .csv file. These features are not validated measures but can be used to visualize activity patterns across the day.

\section{Pre-processing of the actigraphy data}

Actigraphy data are retrieved from devices and saved in a common software database. Sleep intervals are then automatically determined by the built-in actigraphy software platform of the device. Computerized scoring of actigraphy data collected on Philips Respironics Actiwatch models is therefore based on Philips Actiware settings for automatically determining major and minor rest intervals with medium sensitivity (Actiware 6.0.9, Philips Respironics, Murrysville, PA). More than one sleep interval is permitted per day. Philip's default setting for the determination of sleep onset is a period of 10 or more minutes wherein the activity count (AC) is below the threshold of immobility ( $\leq 40$ counts/epoch). The minimum permitted duration for a sleep interval was $15 \mathrm{~min}$, in contrast to the Philips default recommendation of $40 \mathrm{~min}$. There was no set maximum duration for sleep interval duration.
All data were cleaned by one trained researcher who records any adjustments in a standardized cleaning log. A second trained researcher performed a data quality control check on $10 \%$ of the total dataset that is randomly selected to ensure reliability in data cleaning. At a minimum, 72 consecutive hours of data collection with data binned in $\leq 1$-min epochs was required for viable sleep-wake data collected by wrist actigraphy. Days (i.e., 24-h periods) with missing or unreadable data for 4 or more hours were excluded from analysis. Recordings of activity prior to the study start date or after the study end date were excluded from the analysis as well. In instances where actigraphy data was supplemented by sleep diaries or other daily schedule information, the researchers adjusted sleep and wake times in accordance with that information on a study-by-study basis, depending on the source and dependability of the supplementary data. For stand-alone actigraphy data analysis, sleep intervals were only reset in instances when the algorithm missed an instance of wake onset (defined as $\geq 10 \mathrm{~min}$ where $A C \geq 40$ ) or sleep onset (defined as $\geq 10$ min where $\mathrm{AC} \leq 40)$.

\section{Off-wrist determination via the WORK-A transitional dynamics}

The distinction between periods of inactivity due to sedentary behavior versus removal of the device is determined by capacitive sensor automatic off-wrist detection when available. In devices that lack capacitive sensors (i.e., Philips Actiwatch 2.0), off-wrist time is defined by a transitional dynamic approach. Instances where there is an interval of no or little activity $(\mathrm{AC}=0$ during the interval in question or $0<\mathrm{AC}<10 \%$ of the interval duration) but activity is consistently high prior to and following the period of inactivity ( $\geq 10 \mathrm{~min}$ where $\mathrm{AC} \geq 40$ at least $50 \%$ of epochs) are considered off-wrist. Shorter periods of inactivity that are not coded as a sleep interval do not affect data analysis of sleep measures. Hence, for the purposes of sleep-wake determination, periods of off-wrist inactivity are excluded only if the interval has been automatically scored as a sleep interval by the Philips algorithm or if the interval is over $4 \mathrm{~h}$ in length.

\section{Rectifying misrepresentative averages with the WORK-A}

Philips Actiware allows for the export of all interval data (daily intervals, active intervals, rest intervals and sleep intervals) but importantly, key sleep variables (TIB, SOL, TST, WASO, SE) are only computed for sleep intervals. Data can be exported for each individual interval, but there is also the option to export summary statistics averaged across all available intervals. The resulting measure of sleep quantity or quality does not take into account how the number of recorded sleep intervals 
may differ from the number of days that the device was worn though.

Philips Actiware infers the statistics of bedtime, waketime or TIB from interval start time, end time and duration, respectively. The sleep summary statistics do not calculate average start or end times, and sleep interval duration does not account for time in bed prior to falling asleep (i.e., SOL) or time spent in bed after final awakening (called Snoozetime in Actiware). These measures must therefore be added to the sleep interval duration measure to more accurately estimate TIB.

Another issue is the averaging of time to determine habitual bed- or waketime. Actiware defines a day from 12:00-12:00 (noon to noon) rather than 00:00-00:00 (midnight to midnight). While defining a day at midnight is impractical for the purposes of quantifying sleep, defining a day as noon to noon is also not ideal. If a 12$\mathrm{h}$ clock is used, it may be difficult to determine whether time measures refer to AM or PM. A 24-h clock, in contrast, resets at midnight (i.e., 00:00). While it is commonly understood that $00: 03$ on $1 / 2 / 2019$ is later than 23:52 on $1 / 1 / 2019$, averaging these two times mathematically yields a habitual bedtime of 12:37, indicating that the participant normally went to sleep just after noon. For individuals with normal sleep schedules, these errors may be obvious and easy for researchers to catch, but they could skew the interpretation of sleep behavior in service members in an operational context. The WORKA attempts to resolve this issue in two ways. First, prior to calculating averages, the exact time was transformed into a 12 (noon) to 36 (subsequent noon) hour clock by adding $24 \mathrm{~h}$ to each hour between midnight and subsequent noon. In the context of the previous example, 00 : 03 would be recalculated as 24:03, which is numerically greater than 23:52 and yields a more accurate average (23:57 vs. 12:37). Times can be translated back into $24 \mathrm{~h}$ time by subtracting 24:00 from values occurring between midnight (24:00) and subsequent noon (36:00). Second, the WORK-A provides not only average bed- and waketimes but also median bed- and waketimes to provide a measure of habitual sleep schedule, which may be more resilient to outliers.

\section{Comparison of default Actiware summary statistics to those of the WORK-A in ROTC advanced camp cadets}

ROTC cadets during Advanced Camp serve as an ideal candidate group to demonstrate the WORK-A due to the extended duration of the study period ( 31 days), the structured design of camp that enabled testing cadets across a range of mission scenarios in a controlled environment, and the ability to collect self-report data of sleep quality from the cadets. This study was approved by the Walter Reed Army Institute of Research Human Use Review Committee, protocol \#2532C, and was performed in accordance with the ethical standards of the 1964 Declaration of Helsinki. This study was a part of a larger experiment aimed at assessing sleep patterns and sleep quality and their relationships with health and performance. Participants were recruited from cadets undergoing Advanced Camp during routine inprocessing on the first day of camp and gave consent separately for the actigraphy and survey portions of the study. Participants were asked to wear a wrist actigraph (Actiwatch 2.0 Philips Respironics, Murrysville, PA) continuously throughout camp (up to 31 days). Activity data were collected in 30-s epochs, and sleep-wake determination was computed by the Actiware 6.02 scoring algorithm. Participants were also asked to complete the Pittsburgh Sleep Quality Index (PSQI), a one-time selfreport measure of sleep quality from the past month at the end of camp [42].

Summary statistics for key sleep variables were batch exported from Actiware 6.02 prior to WORK-A preprocessing to serve as traditional actigraphy measures of sleep. Sleep-wake determination by the Actiware 6.02 scoring algorithm was not modified by researchers for this traditional actigraphy dataset. Trained researchers then pre-processed each participant's actigraphy data according to the WORK-A standards described above. Cleaned actigraphy data were exported as individual .csv files and compiled using WORK-A computational methods as described above.

\section{Statistical analysis}

Descriptive summary statistics for the traditional and WORK-A actigraphy datasets were computed using Excel 2013 (Microsoft Corp, USA) and SPSS version 25 (IBM Corp, USA). Paired samples $t$-tests were run to compare differences in target sleep variables that existed in both the Actiware sleep summary statistics and the WORK-A. Mean difference scores were computed between habitual bedtime, waketime and sleep duration during camp as recorded by the self-report PSQI, the Actiware summary statistics TST and TIB, and the WORK-A measures TST and TIB. Single sample $t$-tests were conducted to determine if a statistically significant difference existed between mean difference scores. Bland-Altman plots further examined the mean difference between measures of sleep using Excel 2013 and SPSS version 25 .

\section{Results}

Profiling soldier sleep in ROTC cadets via the WORK-A

Actigraphy data were collected from 286 ROTC Advanced Camp cadets (186 men, 68 women and gender unavailable for 32; average age: $22 \pm 3$ years,). Cadets wore the watch for an average of $22 \pm 7$ days during the 31 days of camp. Actiwatches were off-wrist for an 
average of $0.65 \pm 1.38$ days between the time that the cadets first put on and when they last removed the watch. Cadets slept between once and twice per day (DSI: $1.45 \pm 0.25$ ). The median first DSI bedtime was 21:12 \pm 1 : $29 \mathrm{~h}$, and the first DSI wake time was 01:47 $\pm 1: 43 \mathrm{~h}$. The median second DSI bedtime was 01:50 $\pm 2: 02 \mathrm{~h}$, and the second DSI wake time was 04:50 $\pm 1: 51 \mathrm{~h}$.

The results for comparable key sleep variables as measured by Actiware sleep summary statistics and WORKA measures are summarized in Table 2. The Actiware sleep summary statistics were significantly different from all comparable WORK-A measures (all $P \leq 0.001$ ). Key sleep variables were significantly different between the WORK-A all intervals, major, minor and daily (all $P \leq$ 0.05 ) variables except for the WORK-A major and daily measures of WASO $(t=0.55, P=0.58)$. There were no strong correlations $(r>0.5)$ between any WORK-A measures and/or Actiware summary statistics. Estimated average sleep profiles as determined by summary statistics versus the WORK-A are visualized in Fig. 1.

\section{Comparing measures of sleep duration in ROTC cadets via Actiware sleep summary statistics and the WORK-A to self-report measures of sleep}

The results for sleep duration as measured by the PSQI compared to TST and TIB from Actiware summary statistics and the WORK-A are summarized in Fig. 2. Among the 286 ROTC Advanced Camp cadets who wore Actiwatches throughout Advanced Camp, 140 (107 men and 33 women) also completed the PSQI on the last day of camp to provide a self-report of habitual sleep during the training event. In response to the question "During the last month, how many hours of actual sleep did you get at night?", cadets reported $5.62 \pm 0.95 \mathrm{~h}$ $(337 \pm 57 \mathrm{~min})$ per night. Measures of sleep duration from the PSQI, Actiware sleep summary statistics and WORK-A TIB and TST were all significantly different (Fig. 2; all $P \leq 0.05$ ).

Bland-Altman plots of the mean difference of TIB among Actiware data, WORK-A and PSQI are summarized in Fig. 3. One sample $t$ test analysis revealed statistically significant differences, indicating a lack of agreement, between the PSQI sleep duration and both actigraphy-based measures of sleep duration (all $P \leq$ 0.05). Though still significantly different, the smallest mean difference was observed between the PSQI sleep duration and WORK-A major TIB (Fig. 3b; $P=0.006$ ) compared to that between PSQI sleep duration and Actiware TIB (Fig. 3a; $P \leq 0.001$ ) or Actiware TIB and WORK-A major TIB (Fig. 3c; $P \leq 0.001$ ).

Cadets reported an average bedtime of 22:13 $\pm 0: 40 \mathrm{~h}$ for the PSQI question "During the last month, when have you usually gone to bed?" and an average wake time of 04:31 $\pm 0: 47 \mathrm{~h}$. As depicted in Fig. $3 \mathrm{~d}$ and e, the WORK-A major interval median bedtime (22:21 $\pm 1: 30)$ and waketime $(04: 30 \pm 2: 17)$ were in agreement with PSQI-reported bedtime $(P=0.21)$ and waketime $(P=$ $0.68)$, respectively. All other bedtime and wake time parameters were significantly different from those from the PSQI (all $P \leq 0.05$ ).

ROTC cadets failed to meet the minimum sleep duration WORK-A daily TST showed an average of $303 \pm 44 \mathrm{~min}$ of sleep per day. However, cadets failed to meet the specified minimum sleep duration ( $\geq 4 \mathrm{~h} / 24$-h period [41]) for an average of $4 \pm 3$ days during wear time. Only $10 \%$ of the 286 cadets $(n=30)$ consistently met the minimum for all days that they wore the watch. Fifty-seven percent $(57 \% ; n=162)$ of all cadets failed to meet the minimum for between 1 and 4 days, $10 \%$ of cadets $(n=50)$ failed to meet the minimum for between 5 and 10 days and $5 \%$ of all cadets $(n=16)$ failed to meet the minimum for more than 10 days during Advanced Camp.

\section{Discussion}

The WORK-A effectively profile soldier sleep compared to Actiware sleep summary statistics

The advantage of actigraphy as a research tool is its ability to measure sleep accurately in a real-world environment. This ability is particularly important in the operational context where sleep loss could increase the risk for accidents or mission-critical errors. While automated scoring algorithms for sleep-wake determination for actigraphy have been validated against PSG and selfreport, much nuance can be lost through the averaging of these data. The result is a vague estimation of habitual

Table 2 Results for key sleep variables as measured by Philips Actiware and the WORK-A

\begin{tabular}{llllll}
\hline Sleep parameters & $\begin{array}{l}\text { Actiware sleep } \\
\text { summary statistics }\end{array}$ & $\begin{array}{l}\text { WORK-A all intervals } \\
\text { averages }\end{array}$ & $\begin{array}{l}\text { WORK-A daily } \\
\text { averages }\end{array}$ & $\begin{array}{l}\text { WORK-A major } \\
\text { averages }\end{array}$ & $\begin{array}{l}\text { WORK-A minor } \\
\text { averages }\end{array}$ \\
\hline Time in Bed (TIB; min) & $177 \pm 42^{*}$ & $259 \pm 48$ & $366 \pm 44$ & $352 \pm 29$ & $53 \pm 19$ \\
Total sleep time (TST; min) & $161 \pm 39^{*}$ & $214 \pm 44$ & $303 \pm 44$ & $293 \pm 33$ & $38 \pm 16$ \\
Sleep onset latency (SOL; min) & $8 \pm 4^{*}$ & $10 \pm 7$ & $14 \pm 10$ & $12 \pm 9$ & $6 \pm 4$ \\
Wake after sleep onset (WASO; min) & $15 \pm 6^{*}$ & $25 \pm 8$ & $36 \pm 10$ & $35 \pm 15$ & $4 \pm 5$ \\
Sleep efficiency (SE; \%) & $81 \pm 4^{*}$ & $80 \pm 6$ & $83 \pm 5$ & $83 \pm 5$ & $71 \pm 9$ \\
\hline
\end{tabular}

Actiware sleep variables were statistically significant compared to all comparable WORK-A measures ( ${ }^{*}$ represents significance at $P \leq 0.001$, compared against each subsequent column) 


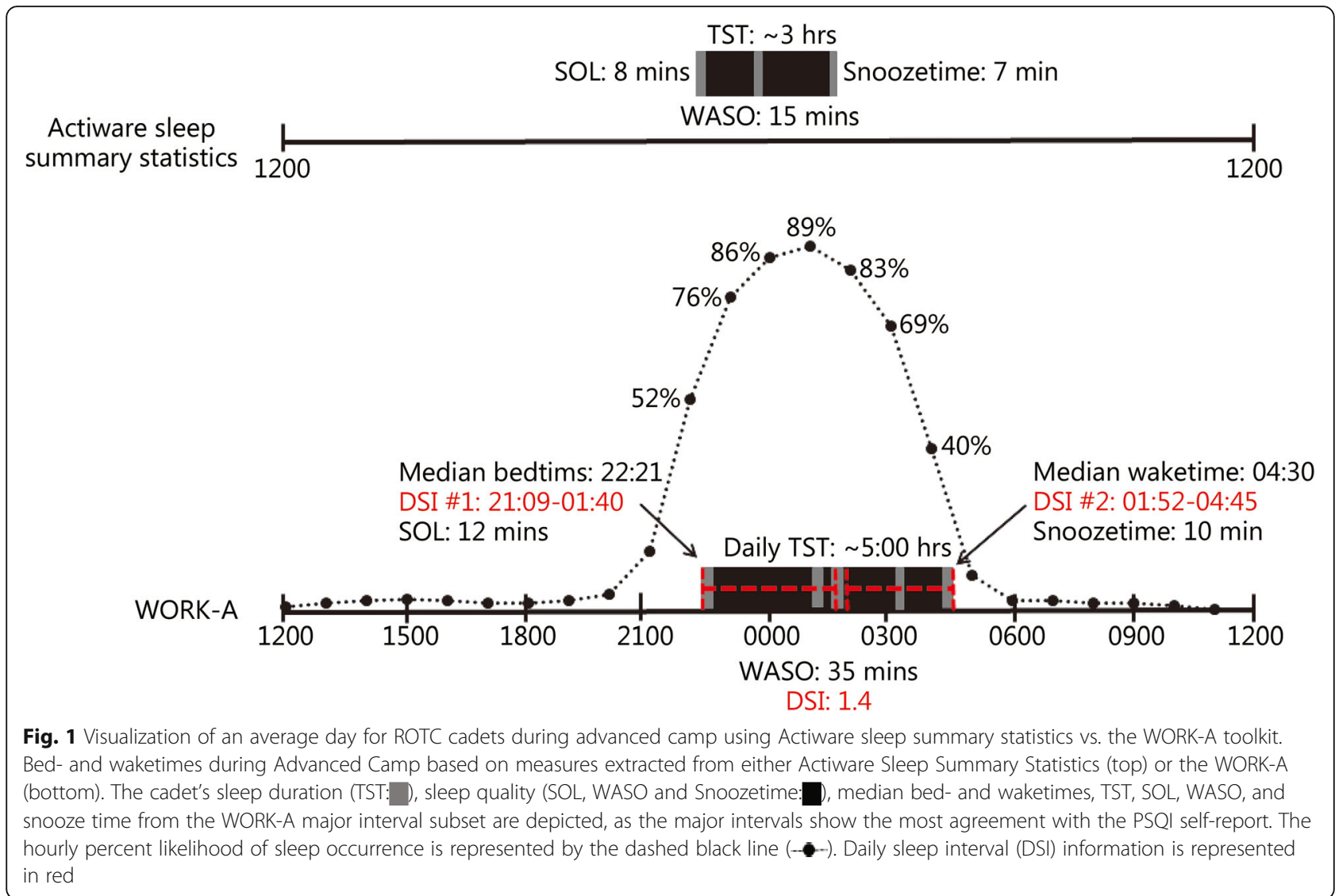

sleep, which may inhibit researchers' ability to interpret erratic sleep patterns, investigate the relationship between specific sleep behaviors and health or performance outcomes, or identify target behaviors for the improvement of sleep duration or quality.

The WORK-A provides researchers with an adaptable range of measures for understanding sleep that do not redefine actigraphy sleep-wake determination. Both Actiware sleep summary statistics and the WORK-A rely on the same set of rules for sleep-wake determination (i.e., those outlined by Philips Actiware). The WORK-A is not a novel computational method for the determination of sleep but a set of procedures for standardized processing of actigraphy data that conforms to Philips sleep-wake determination algorithms (as described in the Methods section) and a toolkit of additional variables that can be calculated from sleep-wake data to provide a more robust understanding of sleep in an operational context.

\section{The pre-processing procedures in the WORK-A may be more suitable for sleep measurements in service members}

In the current study, there were differences in sleep measures due to the WORK-A pre-processing procedures (i.e., verifying that Actiware autoscoring follows sleep-wake determination according to the algorithms' parameters). These cleaning procedures alone accounted for approximately an additional $53 \mathrm{~min}$ of sleep in TST, $83 \mathrm{~min}$ for TIB, $12 \mathrm{~min}$ from SOL and WASO, and a drop in SE from 82 to $79 \%$ compared to actigraphy data that were not processed by a technician. Technician logs for this dataset indicated that there were more instances where the autoscore function failed to catch sleep or wake onset criteria in the actogram than that of data loss including off-wrist inactivity. Therefore, the difference in key sleep variables between the summary statistics and the WORK-A could not be attributed to off-wrist or exclusionary periods. This discrepancy highlights a potential shortcoming in Philips software to reliably determine sleep-wake onset using actigraphy. It should be noted that while Philips Actiware and Actiwatch devices are validated for use in healthy adults, children and sleep-disordered individuals $[20-22,43]$, they were not designed for explicit use in the military operational domain. The preprocessing of actigraphy data to WORK-A standards is time- and labor-intensive, requiring a commitment of approximately $30 \mathrm{~min}$ per participant. Autoscoring would likely be sufficient for populations adhering to a more predictable schedule. 


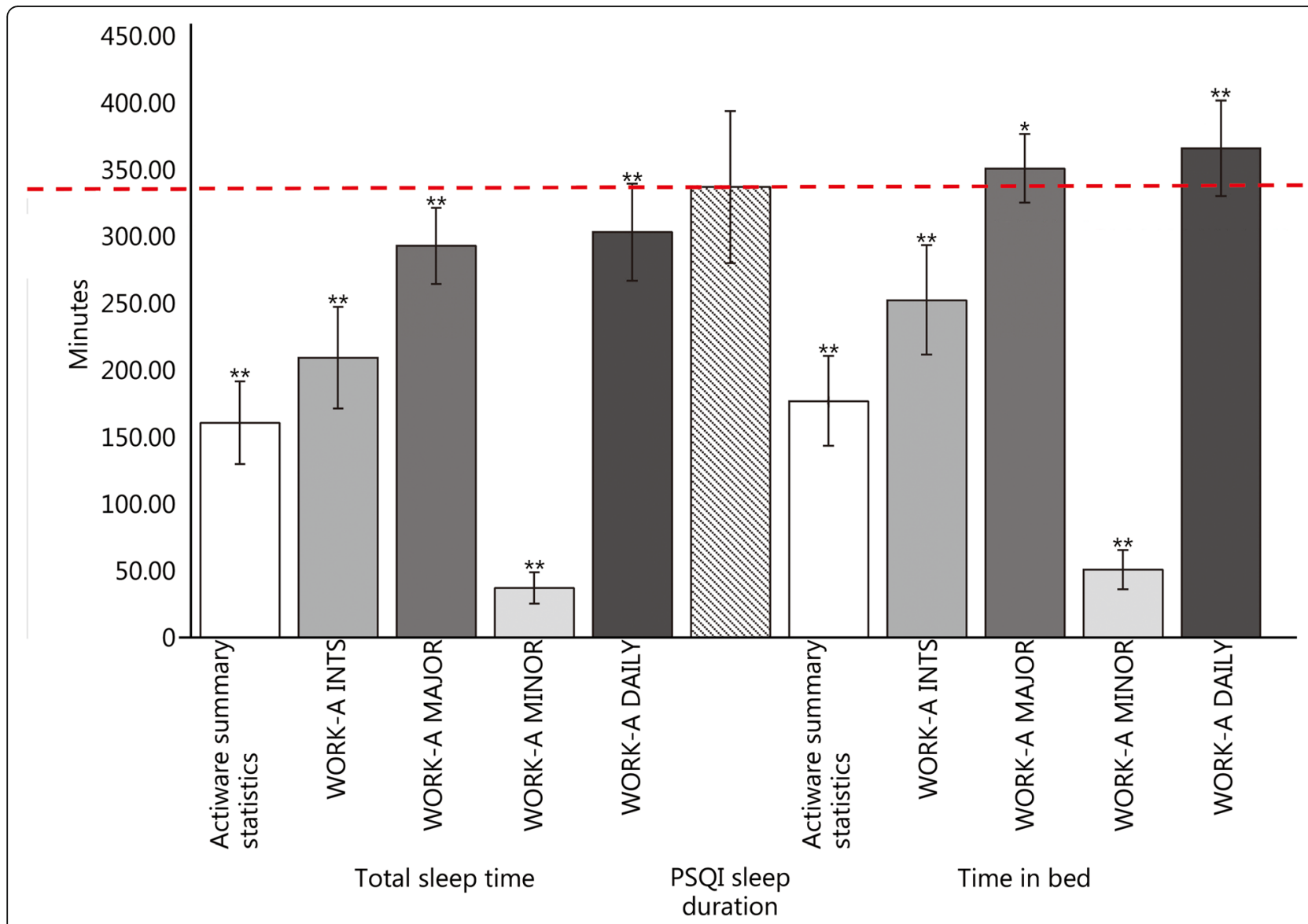

Fig. 2 Average total sleep time and time in bed (in minutes) determined from actigraphy using Actiware versus WORK-A analysis compared to self-report sleep duration. Average TST and TIB as calculated by the Actiware sleep summary statistics $(\square)$, WORK-A all intervals $(\square)$, WORK-A major intervals only $\square$, WORK-A minor intervals only $\square$ and WORK-A daily averages $\square$ in comparison to average self-report nightly sleep duration as measured by the Pittsburgh Sleep Quality Index (PSQI, in minutes. The PSQI sleep duration is represented by the red dashed line $(--) .{ }^{*}$ represents significance at $P \leq 0.05 ;{ }^{* *}$ represents significance at $P \leq 0.001$

\section{Implications for sleep, health and performance} management of ROTC cadets

Although significantly different, the WORK-A major interval measures of sleep came closer to agreement with the PSQI measures of sleep than did the Actiware summary statistics or other WORK-A interval statistics. However, all measures of sleep duration during camp were well below the National Sleep Foundation's recommended $8-10 \mathrm{~h}$ for teenagers and $7-9 \mathrm{~h}$ for young adults [39]. The WORK-A additionally indicated that only $10 \%$ of cadets consistently met the specified minimum of $4 \mathrm{~h}$ of sleep/24-h period during camp. Insufficient sleep is problematic for the health and academic performance of college-aged students in general $[44,45]$, and it constitutes an additional risk to cadets. Insufficient sleep and poor quality sleep may not only negatively impact the immediate performance of ROTC cadets [46], but they can also affect decision-making [47], risk-taking behavior [48], emotion regulation [49] and constructive thinking
[50]. It is reasonable to postulate that adapting to patterns of poor sleep behavior early on could set the stage for detrimental leadership throughout a cadet's military career in a manner that not only affects them but also their future subordinates.

As visualized in Fig. 1, cadets slept mainly at night. However, the hourly analysis of sleep occurrence indicated a 1-5\% likelihood of sleep occurrence during daytime hours, and the minor interval analysis indicated short periods of sleep (i.e., napping) that may not be otherwise have been captured by self-report (the PSQI only asks specifically about nighttime sleep) or traditional analysis of actigraphy data. It should be noted that the ROTC Advanced Camp is highly structured, thus constraining opportunities for sleep. Cadets are chaperoned by US Army soldiers, engage in training events throughout each day and adhere to a structured schedule. Sleep occurrences in other military populations are likely to be less predictable or consolidated 

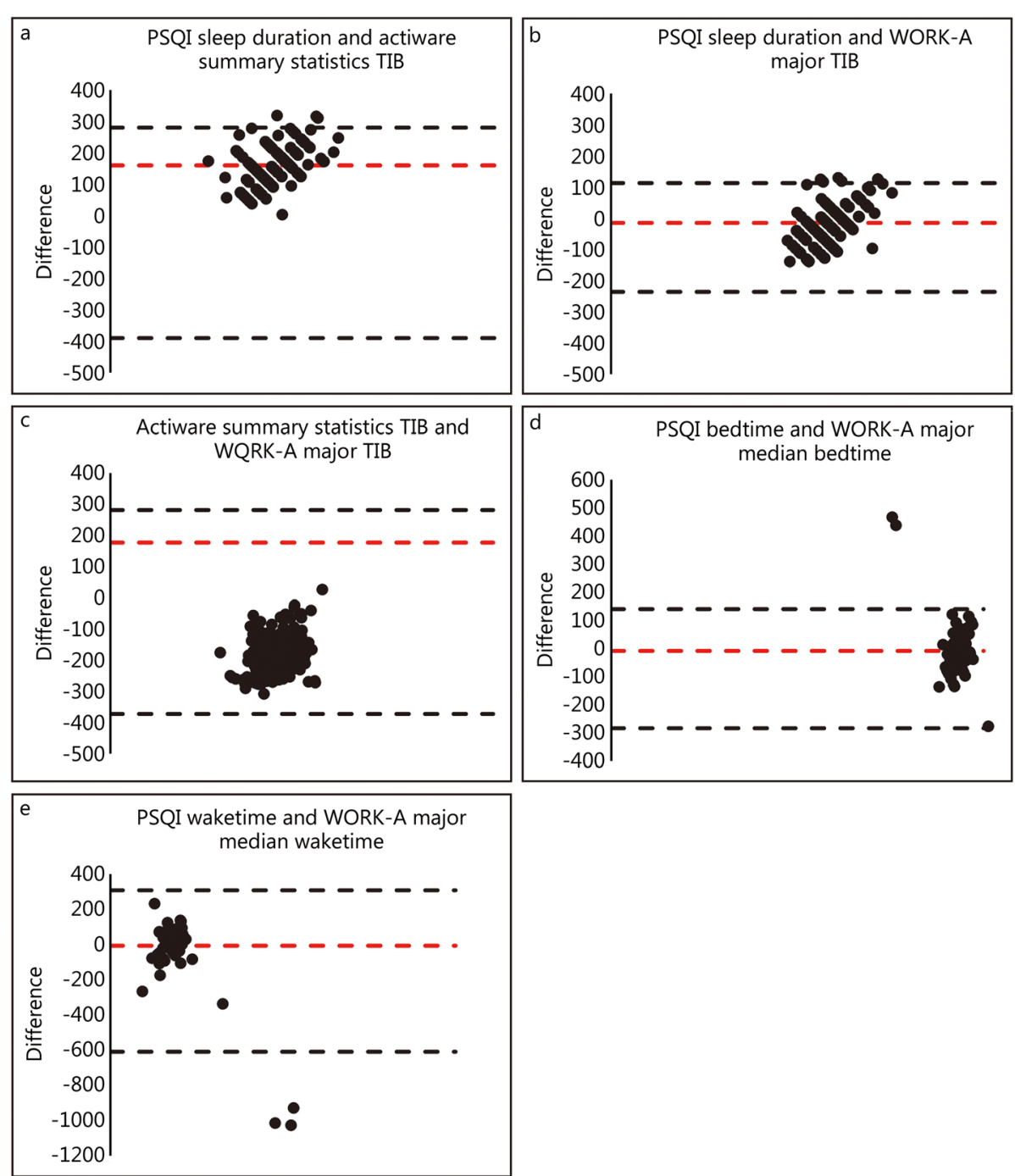

Fig. 3 Bland-Altman plots comparing self-report and actigraphy measures of sleep. Bland-Altman plot of differences ( $y$-axis) between self-report sleep (PSQI) and actigraphy measured sleep vs. the mean of the two measurements ( $x$-axis). Bias is represented by the red dashed line ( -- ), while the upper and lower limits of agreement (LOA) are represented by the black dashed line (- $\mathbf{-})$. $\mathbf{a}$. Agreement between the PSQI sleep duration and Actiware summary statistics TIB $(P \leq 0.001)$. b. Agreement between the PSQI sleep duration and WORK-A major interval TIB $(P=$ 0.006). c. Agreement between the Actiware summary statistics TIB and WORK-A major TIB $(P \leq 0.001)$. $\mathbf{d}$. Agreement between the PSQI reported bedtime and WORK-A major interval median bedtime $(P=0.21)$. e. Agreement between the PSQI reported waketime and WORK-A major interval median waketime $(P=0.68)$

than those of cadets. Napping has been shown to be related to a number of physical and mental health parameters as well as cognitive ability [51-55]. It is possible that taking advantage of brief opportunities for rest could be instrumental in increasing overall sleep duration and improving soldier readiness. Additionally, there are innumerable aspects of a military lifestyle, such as activity patterns [56], diet $[57,58]$, or work schedule [59], which may modulate the relationship between sleep and performance in service members differentially by individual factors such as gender, psychological distress, or chronotype [60-62]. Future analyses will investigate the relationship between sleep, performance and related factors in military groups across the operational domain.

\section{Limitations}

One limitation to profiling sleep in the operational context is the lack of corroborating objective data. More detailed measures of sleep, such as daily sleep diaries, PSG, or direct observation, are often logistically infeasible to obtain given the mission-critical nature of the military domain. Measuring soldier sleep with the current 
limitations to data collection will, however, permit researchers to begin to model sleep patterns in the military domain, opening up the possibility of replicating these conditions in a controlled laboratory environment or closing the gap in our capacity to collect robust data through new technologies or procedures.

An important next step towards validating the WORK-A will be comparing the methodology against PSG in a controlled environment that replicates erratic sleep schedules. To achieve this difficult goal, we plan to collect actigraphy data concurrently with PSG during an in-lab multiple-day sleep restriction protocol. Modified maintenance of wakefulness tests (MWT), which discourage sleep but allow participants to remain asleep if sleep does occur, will be performed throughout the waking period. These conditions should mimic the need to stay vigilant despite insufficient sleep, which is characteristic of sustained military operations and serve as an appropriate proxy for testing the WORK-A in the laboratory.

ROTC cadets in the current study wore Actiwatches throughout Advanced Camp but only completed a onetime habitual self-report of their sleep behavior during Camp (i.e., PSQI). Moreover, the study design allowed participants to consent separately to wear the Actiwatch devices and to complete the surveys. While this design choice yielded greater participation in the actigraphy portion of the study than if cadets were required to complete all aspects of the study, comparisons to selfreport are limited to the subset who agreed to participate in both self-reports of sleep and actigraphy. Not only can self-report be difficult to collect and ultimately subjective, but the PSQI was designed to estimate sleep quality, not to directly measure habitual sleep duration or bed- and waketimes. Future validation of the WORK-A pre-processing procedures for actigraphy will require a comparison against another daily measure of real-world sleep, such as the Pittsburgh Sleep Diary [63]. Moreover, the WORK-A has been demonstrated here in a population of ROTC cadets in a highly controlled environment. Adjustments to the WORK-A may be necessary as it is applied to soldier sleep data in less predictable mission conditions. Another limitation is Actiware sleep summary statistics' inability to calculate average bed- and waketimes, which prevented the comparison of un-processed actigraphy bed-and waketimes to self-report times. This limitation highlights the need for additional computation of variables from actigraphy data, such as those provided by the WORK-A.

\section{Conclusions}

In light of these limitations, the WORK-A represents a step forward towards understanding the role of sleep in soldier readiness. The intent is to apply the methods described above to actigraphy datasets collected from across a wide range of service member groups in simulated combat and deployment operations to investigate how sleep behavior may play a role in vigilance, decision-making, team-level performance, and a number of other military-relevant outcome variables. The WORK-A will also provide a baseline understanding of soldier sleep for the development of military-relevant laboratory sleep studies, provide guidance to military leadership and feedback to service members [64], and ultimately, better protect our nation's soldiers by improving U.S. Army policies and doctrine regarding sleep behavior.

\section{Abbreviations}

AC: Activity count; DSI: Daily sleep intervals; MWT: Maintenance of wakefulness test; ORT: Operational research team; PSG: Polysomnography; PSQI: Pittsburgh Sleep Quality Index; RAR: Rest-activity rhythms; ROTC: Reserve Officer Training Corps; SE: Sleep efficiency; SOL: Sleep onset latency; SSBM: Soldier sleep below minimum; TIB: Time in bed; TST: Total sleep time; WASO: Wake after sleep onset; WORK-A: WRAIR Operational Research Kit-Actigraphy; WRAIR: Walter Reed Army Institute of Research

\section{Acknowledgements}

The material has been reviewed by the Walter Reed Army Institute of Research. There is no objection to its presentation and/or publication. The opinions or assertions contained herein are the private views of the author and are not to be construed as official or as reflecting true views of the Department of the Army or the Department of Defense. The authors would like to acknowledge the Operational Research Team Research Assistants for their hard work collecting and analyzing data as well as their flexibility and dedication to the team. The authors would also like to recognize the ROTC Advanced Camp cadets, cadre and Fort Knox civilian staff for their support and respect towards the research staff during data collection.

\section{Authors' contributions}

JKD, TB and KC were responsible for the development of practice parameters for the pre-processing of actigraphy data, including cleaning procedures and off-wrist determination. JKD and JC were responsible for the selection of WORK-A variables and data analysis. WS was responsible for the ROTC study design. JKD, JC, AM, WS and VFC were responsible for executing the data collection, data management and analysis plan for ROTC data. AM, VFC and WS provided military expertise in identifying knowledge gaps and targets of interest. JKD served as the primary author and project lead for the development of practice parameters, selection of variables, and data analysis plans. WS served as the senior author for this project. The author(s) read and approved the final manuscript.

\section{Funding}

The Department of Defense Military Operational Medicine Research Program (MOMRP) supported this study.

\section{Availability of data and materials}

The datasets generated and/or analyzed during the current study are not publicly available due to the sensitive nature of military training operations and ongoing data analysis but are available from the corresponding author on reasonable request.

\section{Ethics approval and consent to participate}

The investigators have adhered to the policies for protection of human subjects as prescribed in AR 70-25. All study procedures were approved by the Walter Reed Army Institute of Research Institutional Review Board (DoD Assurance: A20126; HHS Federal Wide Assurance: \#00000015; IRB Registration: \#00000794). All participants provided written consent prior to participation in any component of this study. 


\section{Consent for publication}

Not applicable.

\section{Competing interests}

None of the authors have any conflicts of interest to report.

\section{Author details}

'Operational Fatigue and Performance, Institutes for Behavior Resources, Baltimore, MD 21218, USA. ${ }^{2}$ Behavioral Biology Branch, Walter Reed Army Institute of Research, 503 Robert Grant Ave, Silver Spring, MD 20910, USA

${ }^{3}$ Tripler Army Medical Center, Honolulu, HI 96859, USA.

\section{Received: 8 July 2019 Accepted: 12 May 2020}

Published online: 31 May 2020

\section{References}

1. Miller NL, Shattuck LG, Matsangas P. Longitudinal study of sleep patterns of United States military academy cadets. Sleep. 2010;33(12):1623-31.

2. Mysliwiec V, McGraw L, Pierce R, Smith P, Trapp B, Roth BJ. Sleep disorders and associated medical comorbidities in active duty military personnel. Sleep. 2013;36(2):167-74

3. Taylor MK, Hilton SM, Campbell JS, Beckerley SE, Shobe KK, Drummond SP, et al. Prevalence and mental health correlates of sleep disruption among military members serving in a combat zone. Mil Med. 2014;179(7):744-51.

4. Seelig AD, Jacobson IG, Smith B, Hooper TI, Boyko EJ, Gackstetter GD, et al. Sleep patterns before, during, and after deployment to Iraq and Afghanistan. Sleep. 2010;33(12):1615-22.

5. Ohayon MM, Lemoine P, Arnaud-Briant V, Dreyfus M. Prevalence and consequences of sleep disorders in a shift worker population. J Psychosom Res. 2002;53(1):577-83.

6. Wickwire EM, Geiger-Brown J, Scharf SM, Drake CL. Shift work and shift work sleep disorder: clinical and organizational perspectives. Chest. 2017;151(5): 1156-72.

7. Akerstedt T, Wright KP. Sleep loss and fatigue in shift work and shift work disorder. Sleep Med Clin. 2009;4(2):257-71.

8. Sparrow AR, Mollicone DJ, Kan K, Bartels R, Satterfield BC, Riedy SM, et al. Naturalistic field study of the restart break in US commercial motor vehicle drivers: truck driving, sleep, and fatigue. Accid Anal Prev. 2016;93:55-64.

9. Adler AB, GcGurk D, Stetz MC, Bliese PD. Military occupational stressors in garrison, training, and deployed environments. Toronto: Oral presentation at the NIOSH/APA Symposium; 2003.

10. Kripke DF, Mullaney DJ, Messin S, Wyborney VG. Wrist actigraphic measures of sleep and rhythms. Electroencephalogr Clin Neurophysiol. 1978;44(5): 674-6.

11. Brown AC, Smolensky MH, D'Alonzo GE, Redman DP. Actigraphy: a means of assessing circadian patterns in human activity. Chronobiol Int. 1990;7(2): 125-33.

12. Ankers D, Jones SH. Objective assessment of circadian activity and sleep patterns in individuals at behavioural risk of hypomania. J Clin Psychol. 2009:65(10):1071-86.

13. Salvatore P, Ghidini S, Zita G, De Panfilis C, Lambertino S, Maggini C, et al. Circadian activity rhythm abnormalities in ill and recovered bipolar I disorder patients. Bipolar Disord. 2008;10(2):256-65.

14. Vitale JA, Banfi G, Sias M, La Torre A. Athletes' rest-activity circadian rhythm differs in accordance with the sport discipline. Chronobiol Int. 2019;36(4): 578-86.

15. Vitale JA, Negrini F, Rebagliati G, Giacomelli L, Donzelli S, Banfi G. Actigraphy-based sleep parameters and rest-activity circadian rhythm in a young scoliotic patient treated with rigid bracing: a case study. Yale J Biol Med. 2019;92(2):205-12.

16. Smagula SF. Opportunities for clinical applications of rest-activity rhythms in detecting and preventing mood disorders. Curr Opin Psychiatry. 2016;29(6): 389-96.

17. Berry RB, Budhiraja R, Gottlieb DJ, Gozal D, Iber C, Kapur VK, et al. Deliberations of the sleep apnea definitions task force of the American Academy of sleep medicine. Rules for scoring respiratory events in sleep: update of the 2007 AASM manual for the scoring of sleep and associated events. J Clin Sleep Med. 2012;8(5):597-619.

18. Chow CM, Wong SN, Shin M, Maddox RG, Feilds KL, Paxton K, et al. Defining the rest interval associated with the main sleep period in actigraph scoring. Nat Sci Sleep. 2016;8:321-8.
19. Berger AM, Wielgus KK, Young-McCaughan S, Fischer P, Farr L, Lee KA Methodological challenges when using actigraphy in research. J Pain Symptom Manag. 2008;36(2):191-9.

20. Kushida CA, Chang A, Gadkary C, Guilleminault C, Carrillo O, Dement WC Comparison of actigraphic, polysomnographic, and subjective assessment of sleep parameters in sleep-disordered patients. Sleep Med. 2001;2(5):389-96.

21. Lichstein KL, Stone KC, Donaldson J, Nau SD, Soeffing JP, Murray D, et al. Actigraphy validation with insomnia. Sleep. 2006:29(2):232-9.

22. Oakley NR. Validation with polysomnography of the Sleepwatch sleep/wake scoring algorithm used by the Actiwatch activity monitoring system. Bend: Mini Mitter, Camb Neurotechnology; 1997.

23. Sadeh A, Acebo C. The role of actigraphy in sleep medicine. Sleep Med Rev. 2002:6(2):113-24.

24. Littner M, Hirshkowitz M, Kramer M, Kapen S, Anderson WM, Bailey D, et al. Practice parameters for using polysomnography to evaluate insomnia: an update. Sleep. 2003;26(6):754-60.

25. Coffield TG, Tryon WW. Construct validation of actigraphic sleep measures in hospitalized depressed patients. Behav Sleep Med. 2004;2(1):24-40.

26. Morgenthaler T, Alessi C, Friedman L, Owens J, Kapur V, Boehlecke B, et al. Practice parameters for the use of actigraphy in the assessment of sleep and sleep disorders: an update for 2007. Sleep. 2007;30(4):519-29.

27. Sadeh A. The role and validity of actigraphy in sleep medicine: an update. Sleep Med Rev. 2011;15(4):259-67.

28. Calogiuri G, Weydahl A, Carandente F. Methodological issues for studying the rest-activity cycle and sleep disturbances: a chronobiological approach using actigraphy data. Biol Res Nurs. 2013;15(1):5-12.

29. Thomas KA, Burr RL, Spieker S. Maternal and infant activity: analytic approaches for the study of circadian rhythm. Infant Behav Dev. 2015:41:80-7.

30. Camargos EF, Louzada FM, Nobrega OT. Wrist actigraphy for measuring sleep in intervention studies with Alzheimer's disease patients: application, usefulness, and challenges. Sleep Med Rev. 2013;17(6):475-88.

31. Zollman FS, Cyborski C, Duraski SA. Actigraphy for assessment of sleep in traumatic brain injury: case series, review of the literature and proposed criteria for use. Brain Inj. 2010:24(5):748-54.

32. Ancoli-Israel S, Martin JL, Blackwell T, Buenaver L, Liu L, Meltzer LJ, et al. The SBSM guide to actigraphy monitoring: clinical and research applications. Behav Sleep Med. 2015;13(Suppl 1):S4-38.

33. Smith MT, McCrae CS, Cheung J, Martin JL, Harrod CG, Heald JL, et al. Use of actigraphy for the evaluation of sleep disorders and circadian rhythm sleep-wake disorders: an American Academy of sleep medicine clinical practice guideline. J Clin Sleep Med. 2018;14(7):1231-7.

34. Giavarina D. Understanding bland Altman analysis. Biochem Med. 2015 25(2):141-51.

35. Dewitte K, Fierens C, Stockl D, Thienpont LM. Application of the blandAltman plot for interpretation of method-comparison studies: a critical investigation of its practice. Clin Chem. 2002:48(5):799-801.

36. Fischer D, Vetter $C$, Roenneberg T. A novel method to visualise and quantify circadian misalignment. Sci Rep. 2016;6:38601

37. Dashti HS, Zuurbier LA, de Jonge E, Voortman T, Jacques PF, Lamon-Fava S, et al. Actigraphic sleep fragmentation, efficiency and duration associate with dietary intake in the Rotterdam study. J Sleep Res. 2016:25(4):404-11.

38. Leeder J, Glaister M, Pizzoferro K, Dawson J, Pedlar C. Sleep duration and quality in elite athletes measured using wristwatch actigraphy. J Sports Sci. 2012;30(6):541-5.

39. Hirshkowitz M, Whiton K, Albert SM, Alessi C, Bruni O, DonCarlos L, et al. National Sleep Foundation's updated sleep duration recommendations: final report. Sleep Health. 2015;1(4):233-43.

40. Headquarters Department of the Army. Field manual 6.22.5: Combat and operational stress control manual for leaders and soldiers. Washington, DC; 2009.

41. Headquarters Department of the Army. Leader's manual for combat stress control (FM 22-51). 1994

42. Buysse DJ, Reynolds CF 3rd, Monk TH, Berman SR, Kupfer DJ. The Pittsburgh sleep quality index: a new instrument for psychiatric practice and research. Psychiatry Res. 1989;28(2):193-213.

43. Puyau MR, Adolph AL, Vohra FA, Butte NF. Validation and calibration of physical activity monitors in children. Obes Res. 2002;10(3):150-7.

44. Forquer LM, Camden AE, Gabriau KM, Johnson CM. Sleep patterns of college students at a public university. J Am Coll Heal. 2008;56(5):563-5.

45. Owens H, Christian B, Polivka B. Sleep behaviors in traditional-age college students: a state of the science review with implications for practice. J Am Assoc Nurse Pract. 2017;29(11):695-703. 
46. Ritland BM, Simonelli G, Gentili RJ, Smith JC, He X, Oh H, et al. Sleep health and its association with performance and motivation in tactical athletes enrolled in the reserve Officers' training corps. Sleep Health. 2019;5(3):309-14.

47. Harrison $Y$, Horne JA. The impact of sleep deprivation on decision making: a review. J Exp Psychol Appl. 2000;6(3):236-49.

48. Womack SD, Hook JN, Reyna SH, Ramos M. Sleep loss and risk-taking behavior: a review of the literature. Behav Sleep Med. 2013;11(5):343-59.

49. Palmer CA, Alfano CA. Sleep and emotion regulation: an organizing, integrative review. Sleep Med Rev. 2017;31:6-16.

50. Killgore WD, Kahn-Greene ET, Lipizzi EL, Newman RA, Kamimori GH, Balkin TJ. Sleep deprivation reduces perceived emotional intelligence and constructive thinking skills. Sleep Med. 2008;9(5):517-26.

51. Davy J, Gobel M. The effects of extended nap periods on cognitive, physiological and subjective responses under simulated night shift conditions. Chronobiol Int. 2018:35(2):169-87.

52. Ficca G, Axelsson J, Mollicone DJ, Muto V, Vitiello MV. Naps, cognition and performance. Sleep Med Rev. 2010;14(4):249-58.

53. Mantua J, Spencer RMC. Exploring the nap paradox: are mid-day sleep bouts a friend or foe? Sleep Med. 2017;37:88-97.

54. Devine JK, Wolf JM. Integrating nap and night-time sleep into sleep patterns reveals differential links to health-relevant outcomes. I Sleep Res. 2016;25(2):225-33

55. Leger D, Torres MJ, Bayon V, Hercberg S, Galan P, Chennaoui M, et al. The association between physical and mental chronic conditions and napping. Sci Rep. 2019;9(1):1795

56. Bernhardt KA, Kelley AM, Feltman KA, Curry IP. Rest and activity patterns of army aviators in routine and operational training environments. Aerosp Med Hum Perform. 2019;90(1):48-52.

57. St-Onge MP, Mikic A, Pietrolungo CE. Effects of diet on sleep quality. Adv Nutr. 2016;7(5):938-49.

58. Farina EK, Taylor JC, Means GE, Murphy NE, Pasiakos SM, Lieberman HR, et al. Effects of deployment on diet quality and nutritional status markers of elite U.S. Army special operations forces soldiers. Nutr J. 2017;16(1):41.

59. Caldwell JL, Gilreath SR. Work and sleep hours of U.S. Army aviation personnel working reverse cycle. Mil Med. 2001;166(2):159-66.

60. Vitale JA, Weydahl A. Chronotype, physical activity, and sport performance: a systematic review. Sports Med. 2017:47(9):1859-68.

61. Saidi O, Dore E, Maso F, Mack-Inocentio D, Walrand S, Pereira B, et al. Acute effect of an intensified exercise program on subsequent sleep, dietary intake, and performance in junior rugby players. Eur J Appl Physiol. 2019; 119(9):2075-82.

62. Hansen LP, Kinskey C, Koffel E, Polusny M, Ferguson J, Schmer-Galunder S, et al. Sleep patterns and problems among Army National Guard Soldiers. Mil Med. 2018;183(11-12):e396-401.

63. Monk TH, Reynolds CF 3rd, Kupfer DJ, Buysse DJ, Coble PA, Hayes AJ, et al. The Pittsburgh sleep diary. J Sleep Res. 1994;3:111-20.

64. Adler AB, Gunia BC, Bliese PD, Kim PY, LoPresti ML. Using actigraphy feedback to improve sleep in soldiers: an exploratory trial. Sleep Health. 2017;3(2):126-31.

\section{Ready to submit your research? Choose BMC and benefit from:}

- fast, convenient online submission

- thorough peer review by experienced researchers in your field

- rapid publication on acceptance

- support for research data, including large and complex data types

- gold Open Access which fosters wider collaboration and increased citations

- maximum visibility for your research: over $100 \mathrm{M}$ website views per year

At BMC, research is always in progress.

Learn more biomedcentral.com/submissions 